\title{
anu \\ EOB-MR Based Radiomics Analysis to Assess Clinical Outcomes following Liver Resection in Colorectal Liver Metastases
}

Vincenza Granata ${ }^{1, *(D)}$, Roberta Fusco ${ }^{2}$, Federica De Muzio ${ }^{3}$, Carmen Cutolo ${ }^{4}$, Sergio Venanzio Setola ${ }^{1}$, Federica Dell'Aversana ${ }^{5}$, Alessandro Ottaiano ${ }^{6}{ }^{(D}$, Guglielmo Nasti ${ }^{6}$, Roberta Grassi ${ }^{5}$, Vincenzo Pilone ${ }^{4}$, Vittorio Miele ${ }^{7,8} \mathbb{D}$, Maria Chiara Brunese ${ }^{3}$, Fabiana Tatangelo ${ }^{9} \mathbb{D}$, Francesco Izzo ${ }^{10}$ (D) and Antonella Petrillo ${ }^{1} \mathbb{D}$

check for

updates

Citation: Granata, V.; Fusco, R.; De

Muzio, F.; Cutolo, C.; Setola, S.V.;

Dell'Aversana, F.; Ottaiano, A.; Nasti,

G.; Grassi, R.; Pilone, V.; et al

EOB-MR Based Radiomics Analysis

to Assess Clinical Outcomes

following Liver Resection in

Colorectal Liver Metastases. Cancers

2022, 14, 1239. https://doi.org/

10.3390/cancers14051239

Academic Editor: Éric Chastre

Received: 18 January 2022

Accepted: 25 February 2022

Published: 27 February 2022

Publisher's Note: MDPI stays neutral with regard to jurisdictional claims in published maps and institutional affiliations.

Copyright: (C) 2022 by the authors. Licensee MDPI, Basel, Switzerland. This article is an open access article distributed under the terms and conditions of the Creative Commons Attribution (CC BY) license (https:// creativecommons.org/licenses/by/ $4.0 /)$
1 Division of Radiology, Istituto Nazionale Tumori IRCCS Fondazione Pascale-IRCCS di Napoli, 80131 Napoli, Italy; s.setola@istitutotumori.na.it (S.V.S.); a.petrillo@istitutotumori.na.it (A.P.)

2 Medical Oncology Division, Igea SpA, 41012 Carpi, Italy; r.fusco@igeamedical.com

3 Diagnostic Imaging Section, Department of Medical and Surgical Sciences \& Neurosciences, University of Molise, 86100 Campobasso, Italy; demuziofederica@gmail.com (F.D.M.); m.brunese@studenti.unimol.it (M.C.B.)

4 Department of Medicine, Surgery and Dentistry, University of Salerno, 84084 Fisciano, Italy; carmencutolo@hotmail.it (C.C.); vpilone@unisa.it (V.P.)

5 Division of Radiology, Università degli Studi della Campania Luigi Vanvitelli, 81100 Caserta, Italy; federica.dellaversana@unicampania.it (F.D.); roberta.grassi@policliniconapoli.it (R.G.)

6 Division of Abdominal Oncology, Istituto Nazionale Tumori IRCCS Fondazione Pascale-IRCCS di Napoli, 80131 Napoli, Italy; a.ottaiano@istitutotumori.na.it (A.O.); g.nasti@istitutotumori.na.it (G.N.)

7 Division of Radiology, Azienda Ospedaliera Universitaria Careggi, 50134 Firenze, Italy; vmiele@sirm.org

8 Italian Society of Medical and Interventional Radiology (SIRM), SIRM Foundation, 20122 Milan, Italy

9 Division of Pathology, Istituto Nazionale Tumori IRCCS Fondazione Pascale-IRCCS di Napoli, 80131 Napoli, Italy; f.tatangelo@istitutotumori.na.it

10 Division of Epatobiliary Surgical Oncology, Istituto Nazionale Tumori IRCCS Fondazione Pascale-IRCCS di Napoli, 80131 Napoli, Italy; f.izzo@istitutotumori.na.it

* Correspondence: v.granata@istitutotumori.na.it

Simple Summary: The aim of this study was to assess the efficacy of radiomics features obtained by EOB-MRI phase in order to predict clinical outcomes following liver resection in Colorectal Liver Metastases Patients, and evaluate recurrence, mutational status, pathological characteristic (mucinous) and surgical resection margin. Ours results confirmed the capacity of radiomics to identify, as biomarkers, several prognostic features that could affect the treatment choice in patients with liver metastases, in order to obtain a more personalized approach. These results were confirmed by external validation dataset. We obtained a good performance considering the single textural significant metric in the identification of front of tumor growth (expansive versus infiltrative) and tumor budding (high grade versus low grade or absent), in the recognition of mucinous type and in the detection of recurrences.

Abstract: The aim of this study was to assess the efficacy of radiomics features obtained by EOB-MRI phase in order to predict clinical outcomes following liver resection in Colorectal Liver Metastases Patients, and evaluate recurrence, mutational status, pathological characteristic (mucinous) and surgical resection margin. This retrospective analysis was approved by the local Ethical Committee board of National Cancer of Naples, IRCCS "Fondazione Pascale". Radiological databases were interrogated from January 2018 to May 2021 in order to select patients with liver metastases with pathological proof and EOB-MRI study in pre-surgical setting. The cohort of patients included a training set (51 patients with 61 years of median age and 121 liver metastases) and an external validation set (30 patients with single lesion with 60 years of median age). For each segmented volume of interest by 2 expert radiologists, 851 radiomics features were extracted as median values using PyRadiomics. non-parametric test, intraclass correlation, receiver operating characteristic (ROC) analysis, linear regression modelling and pattern recognition methods (support vector machine (SVM), k-nearest neighbors (KNN), artificial neural network (NNET), and decision tree (DT)) were 
considered. The best predictor to discriminate expansive versus infiltrative front of tumor growth was HLH_glcm_MaximumProbability extraxted on VIBE_FA30 with an accuracy of $84 \%$, a sensitivity of $83 \%$, and a specificity of $82 \%$. The best predictor to discriminate tumor budding was Inverse Variance obtained by the original GLCM matrix extraxted on VIBE_FA30 with an accuracy of 89\%, a sensitivity of $96 \%$ and a specificity of $65 \%$. The best predictor to differentiate the mucinous type of tumor was the HHL_glszm_ZoneVariance extraxted on VIBE_FA30 with an accuracy of 85\%, a sensitivity of $46 \%$ and a specificity of $95 \%$. The best predictor to identify tumor recurrence was the LHL_glcm_Correlation extraxted on VIBE_FA30 with an accuracy of $86 \%$, a sensitivity of $52 \%$ and a specificity of $97 \%$. The best linear regression model was obtained in the identification of the tumor growth front considering the height textural significant metrics by VIBE_FA10 (an accuracy of $89 \%$; sensitivity of $93 \%$ and a specificity of $82 \%$ ). Considering significant texture metrics tested with pattern recognition approaches, the best performance for each outcome was reached by a KNN in the identification of recurrence with the 3 textural significant features extracted by VIBE_FA10 (AUC of $91 \%$, an accuracy of $93 \%$; sensitivity of $99 \%$ and a specificity of $77 \%$ ). Ours results confirmed the capacity of radiomics to identify as biomarkers, several prognostic features that could affect the treatment choice in patients with liver metastases, in order to obtain a more personalized approach.

Keywords: Liver metastasis; Magnetic Resonance Imaging; artificial intelligence; radiomics

\section{Introduction}

Radiomics is a rapidly evolving field of research concerned with the extraction of quantitative metrics - the so-called radiomics features-within medical images. Radiomic features capture tissue and lesion characteristics such as heterogeneity and shape and may, alone or in combination with demographic, histologic, genomic, or proteomic data, be used for clinical problem solving. In oncology, the assessment of tissue heterogeneity is of particular interest; genomic analyses have demonstrated that the degree of tumor heterogeneity is a prognostic determinant of survival and an obstacle to cancer control. Studies have demonstrated that radiomics features are strongly correlated with heterogeneity indices at the cellular level [1-8]. Therefore, that Radiomics could support cancer detection, diagnosis, evaluation of prognosis and response to treatment, so as could supervise disease status [9-14]. Using standard of care images that are usually obtained in a clinical setting, Radiomics analysis is a cost-effective and highly feasible implement for clinical decision support, providing prognostic and/or predictive biomarkers which enables a fast, low-cost, and repeatable tool for longitudinal monitoring [15-20]. Even though individual features may correlate with genomic data, so-called radiogenomics, or clinical outcomes, the impact of radiomics is increased when the data are processed using machine learning techniques. Nowadays, several studies have assessed the role of radiogenomics in hepatocellular carcinoma, but only a few have examined liver metastases [1-3].

During the work-up of patients with liver metastases, imaging plays an important role, since it enables one to estimate the number and sites of lesions, to assess the resectability, and to evaluate the response to treatment and drug toxicities [21-25]. Though computed tomography (CT) is routinely used for primary staging and disease surveillance, Magnetic Resonance imaging (MRI) is a valuable diagnostic technique in oncologic settings since it allows one to assess morphological and functional data [21-24]. Moreover, several liver-specific contrast agents have been introduced to improve lesions detection and characterization. Gadobenate dimeglumine (Gd-BOPTA) and gadolinium ethoxybenzyl diethylenetriamine pentaacetic acid (Gd-EOB-DTPA) allow one to obtain data on the lesions vascularization during the different phases of contrast study and functional data in the delayed, hepatobiliary phase (EOB-phase).

In this context, the possibility to correlate radiomics parameters obtained by MRI studies to recurrences, mutational status, pathological characteristic (mucinous and tumor budding), and surgical resection margin offers notable advantages over qualitative 
imaging assessment, allowing a better patient selection for cancer therapy, treatment response prediction, and discrimination of favorable subsets of patients from those with poor prognosis. In the present study, we assessed the efficacy of radiomics features obtained by EOB-MRI phase to predict clinical outcomes following liver resection in Colorectal Liver Metastases Patients.

\section{Materials and Methods}

\subsection{Dataset Characteristics}

This study aligned with National appropriate guidelines and procedures. The National Cancer Institute of Naples Ethical Committee board approved this retrospective study, renouncing the need for informed patient consent given the study nature.

Radiological archive was evaluated from January 2018 to May 2021 in order to choose patients with: (1) liver metastases with pathological proof; (2) EOB-MRI study in presurgical setting after neoadjuvant chemotherapy; (3) MR images of high quality; (4) a followup CT scan of at least six months after surgery. The exclusion criteria were: (1) discordance among the imaging diagnosis and the pathologically ones, (2) no EOB-MRI phase studies; (3) no high-quality MR images.

The cohort of patients included a training set and an external validation set. The internal training set consisted of 51 patients ( 33 women and $18 \mathrm{men}$ ) with a median age of 61 years (range 35-82 years) and 121 liver metastases. The validation cohort consisted of 30 patients with single lesion (10 women and 20 men) with 60 years of median age (range $40-78$ years). The external validation patient dataset was provided by "Careggi Hospital", Florence, Italy.

No liver metastases identified by EOB-MRI during the period investigated in this retrospective study were pathologically confirmed and thus not included in the study.

The patient characteristics are summarized in Table 1.

\subsection{MR Imaging Protocol}

MR studies were performed with 2 1.5T MR tomographs: a Magnetom Symphony (Siemens, Erlangen, Germany) and Magnetom Aera (Siemens). The MRI images were acquired before and after an intravenous (IV) contrast agent (CA) injection.

In this study, a radiomics features extraction was made on volumetric interpolated breath-hold examination (VIBE) T1-weighted SPAIR with controlled respiration used to acquire images after IV CA injection with a liver-specific CA $(0.1 \mathrm{~mL} / \mathrm{kg}$ of Gd-EOBBPTA-Primovist, Bayer Schering Pharma, Berlin, Germany). The VIBE T1-W sequence was acquired with 2 different flip angles (10 and 30 degrees). A power injector (Spectris Solaris ${ }^{\circledR}$ EP MR, MEDRAD Inc., Indianola, IA, USA) was used to administrate the CA at an infusion rate of $2 \mathrm{~mL} / \mathrm{s}$, as descripted in our previous studies [26,27]. Table 2 reports MR Sequence parameters.

\subsection{Image Processing}

Regions of interest (ROIs) were manually drawn slice-by-slice by 2 expert radiologists with 22 and 15 years of abdominal imaging experience, respectively; first separately and then together and in accordance with each other, annotating all of the slices of the lesions. The segmentation was performed on arterial phase of VIBE T1-W images for both sequences acquired using 10 and 30 degrees of flip angle. For these reasons, we performed the analysis on 2 sequence VIBE_FA10 (VIBE T1-W images with flip angle $10^{\circ}$ ) and VIBE_FA30 (VIBE T1$\mathrm{W}$ images with flip angle $30^{\circ}$ ). Manual definition of the ROIs was made using segmentation tool of 3DSlicer (https:/ / www.slicer.org/; accessed on 21 December 2021). 
Table 1. Characteristics of the study population (81 patients).

\begin{tabular}{|c|c|}
\hline Patient Description & Numbers $(\%) /$ Range \\
\hline \multirow{2}{*}{ Gender } & Men $53(65.4 \%)$ \\
\hline & Women $28(34.6 \%)$ \\
\hline Age & 61 years; range: $35-82$ years \\
\hline \multicolumn{2}{|l|}{ Primary Cancer Site } \\
\hline Colon & $52(64.2 \%)$ \\
\hline Rectum & $29(35.8 \%)$ \\
\hline Prior Chemotherapy & $81(100 \%)$ \\
\hline \multicolumn{2}{|l|}{ Hepatic Metastases Description } \\
\hline Patients with single nodule & $52(64.2 \%)$ \\
\hline Patients with multiple nodules & $29(35.8 \%) /$ range: $2-13$ metastases \\
\hline Nodule size (mm) & mean size $36.4 \mathrm{~mm}$; range $7-58 \mathrm{~mm}$ \\
\hline \multicolumn{2}{|l|}{ Front of Tumor Growth } \\
\hline expansive & $30(37.0 \%)$ \\
\hline infiltrative & $51(63.0 \%)$ \\
\hline \multicolumn{2}{|l|}{ Tumor Budding } \\
\hline Absent & $12(14.8 \%)$ \\
\hline Low grade & $14(17.3 \%)$ \\
\hline High grade & $55(67.9 \%)$ \\
\hline Mucinous Carcinoma & $25(30.9 \%)$ \\
\hline Recurrence & $19(23.5 \%)$ \\
\hline RAS Mutation & $42(51.9 \%)$ \\
\hline
\end{tabular}

Table 2. MR Sequence parameters.

\begin{tabular}{|c|c|c|c|c|c|c|}
\hline Sequence & Orientation & $\begin{array}{c}\text { TR/TE/FA } \\
\text { (ms/ms/deg.) }\end{array}$ & $\underset{(\mathrm{min})}{\mathrm{AT}}$ & $\begin{array}{l}\text { Acquisition } \\
\text { Matrix }\end{array}$ & ST/Gap (mm) & FS \\
\hline Trufisp T2-W & Coronal & $4.30 / 2.15 / 80$ & 0.46 & $512 \times 512$ & $4 / 0$ & without \\
\hline HASTE T2-W & Axial & $1500 / 90 / 170$ & 0.36 & $320 \times 320$ & $5 / 0$ & $\begin{array}{l}\text { without and } \\
\text { with (SPAIR) }\end{array}$ \\
\hline HASTE T2w & Coronal & $1500 / 92 / 170$ & 0.38 & $320 \times 320$ & $5 / 0$ & without \\
\hline In-Out phase T1-W & Axial & $160 / 2.35 / 70$ & 0.33 & $256 \times 192$ & $5 / 0$ & without \\
\hline $\begin{array}{c}\text { VIBE } \\
\text { T1-W_FA10 }\end{array}$ & Axial & $4.80 / 1.76 / 10$ & 0.18 & $320 \times 260$ & $3 / 0$ & with (SPAIR) \\
\hline $\begin{array}{c}\text { VIBE } \\
\text { T1-W_FA30 }\end{array}$ & Axial & $4.80 / 1.76 / 30$ & 0.18 & $320 \times 260$ & $3 / 0$ & with (SPAIR) \\
\hline
\end{tabular}

Note: $\mathrm{W}=$ Weighted, $\mathrm{TR}=$ Repetition time, $\mathrm{TE}=$ Echo time, $\mathrm{FA}=$ Flip angle, $\mathrm{AT}=$ Acquisition time SPAIR = Spectral Adiabatic Inversion Recovery, VIBE = Volumetric interpolated breath hold examination, HASTE $=$ HASTE $=$ Half-Fourier-Acquired Single-shot Turbo spin Echo .

\subsection{MRI Post-Processing with Pyradiomics Tool}

For each volume of interest, 851 radiomics features were extracted as median values using open-source PyRadiomics python package [28].

We used wavelet filtering (LLH, LHL, LHH, LLL, HLL, HLH, HHL, HHH high (H) or low (L) -pass filters along the $\mathrm{X}$ and $\mathrm{Y}$ axis and a $\mathrm{Z}$-axis) to six different matrices: 
- $\quad$ First Order (FIRST ORDER): describes the individual values of voxels obtained as a result of ROI cropping. These are generally histogram-based properties (energy, entropy, kurtosis, skewness);

- $\quad$ Shape based features both 2D and 3D measures;

- Gray Level Co-occurrence Matrix (GLCM): calculates how often the same and similar pixel values come together in an image and records statistical measurements according to this matrix. These resulting values numerically characterize the texture of the image;

- Gray Level Run Length Matrix (GLRLM): Defined as the number of homogeneous consecutive pixels with the same gray tone and quantifies the gray-level studies;

- Gray Level Size Zone Matrix (GLSZM): Properties based on this matrix assign voxel counts according to the logic of measuring gray-level regions in an image;

- $\quad$ Neighboring Gray Tone Difference Matrix (NGTDM): Digitization of textures obtained from filtered images and their fractal properties;

- $\quad$ Gray Level Dependence Matrix (GLDM): Number of bound voxels at x distance from the central voxel;

The extracted features are in compliance with feature definitions as described by the Imaging Biomarker Standardization Initiative (IBSI) [29] and as reported in (https: / / readthedocs.org/projects/pyradiomics/downloads/; accessed on 21 December 2021).

Median values of radiomics features were considered for each segmented volume of interest.

A graphical representation of the radiomics process and of the extracted features has been reported in Figure 1. However, Radiomics involves 3D qualitative and quantitative high throughput extraction of digital imaging data that cannot be represented as an image.

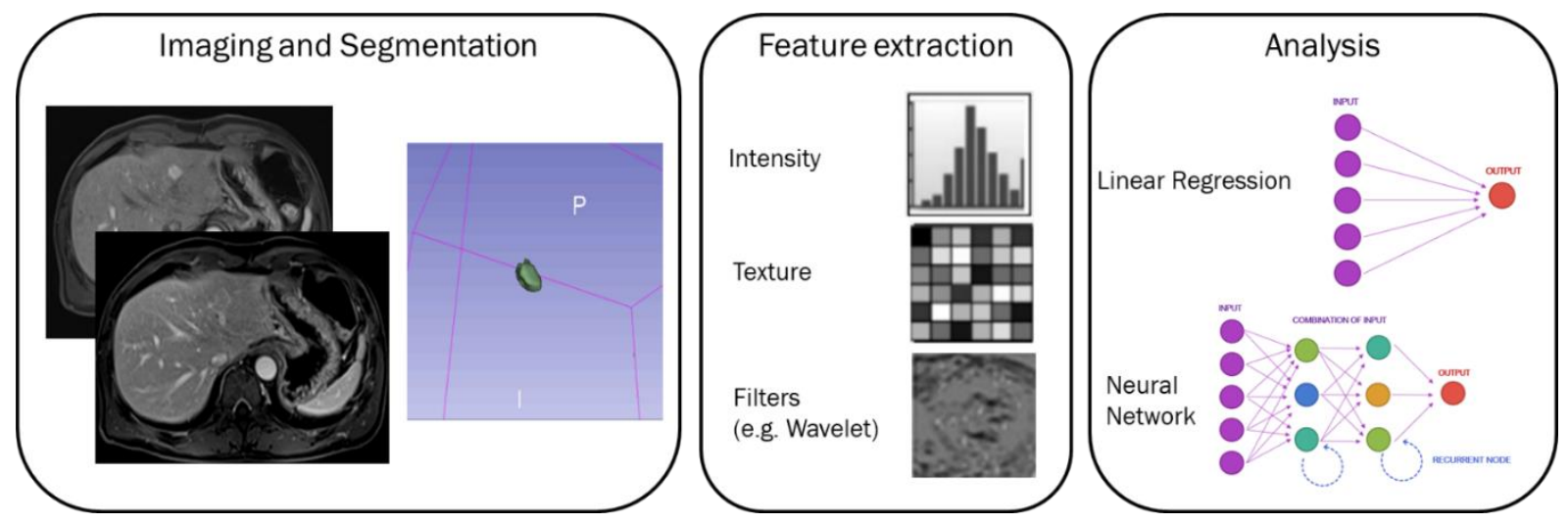

Figure 1. A graphical representation of the radiomics process and of the extracted features.

Radiomics analysis was performed blinded to the clinical and pathological data in pre-surgical setting after neoadjuvant chemotherapy. No registration techniques to reduce movements artefacts were applied, however, the use of median value of extracted metrics allows one to reduce the influence of artefacts.

\subsection{Statistical Analysis}

Statistical analysis includes both univariate and multivariate approaches performed considering a per-lesion analysis. The statistical analyses were performed using the Statistics and Machine Toolbox of MATLAB R2021b (MathWorks, Natick, MA, USA).

\subsection{Univariate Analysis}

The assessment of observer variability was performed by calculating the intraclass correlation coefficient.

A non-parametric Kruskal-Wallis test was performed to identify differences statistically significant among clinical parameters and radiomics metrics of two groups (front of tumor 
growth: expansive versus infiltrative; tumor budding: high grade versus low grade or absent; mucinous type and presence of recurrence).

Receiver operating characteristic (ROC) analysis was performed using the Youden index to calculate the optimal cut-off for each metric and then the area under the ROC curve (AUC), sensitivity, specificity, positive predictive value (PPV), negative predictive value (NPV) and accuracy.

A $p$ value $<0.05$ was considered as significant.

\subsection{Multivariate Analysis}

A multivariate analysis was performed in order to identify the combinations of variables which best predict the outcomes: (1) front of tumor growth: expansive versus infiltrative; (2) tumor budding: high grade versus low grade or absent; (3) mucinous type; (4) presence of recurrence.

Given the high number of textural features, a first selection of variables was made based on the results obtained from the univariate analysis: significant at nonparametric Kruskal-Wallis test and with an accuracy $\geq 75 \%$. A linear regression modelling was used to assess the best linear combination of significant textural features for each outcome. The linear regression model was used to assess the accuracy of linear combination and ROC analysis with Youden index was used to identify the optimal cut-off value. Considering the optimal cut-off value, we reported accuracy, sensitivity, specificity, PPV and NPV.

Pattern recognition techniques including support vector machine (SVM), k-nearest neighbors (KNN), artificial neural network (NNET), and decision tree (DT) were performed to calculate the diagnostic performance considering all of the features and/or a subset of features after a feature selection approach [30]. The best model was identified calculating the highest area under ROC curve and highest accuracy. Each classifier was trained with a 10-k fold cross validation; therefore, median values of AUC, accuracy, sensitivity, and specificity were calculated. Moreover, an external validation cohort was used to validate the findings of the best classifier.

\section{Results}

\subsection{Univariate Analysis Findings}

The median value of intraclass correlation coefficients for features was 0.92 (range $0.86-0.96)$. The size of the lesion did not affect the extracted metrics ( $p$-value $>0.05$ at the Kruskal-Wallis test performed between the 2 groups: lesions $<2 \mathrm{~cm}$ and lesions $\geq 2 \mathrm{~cm}$ ). In addition, the RAS mutational status did not affect the extracted metrics ( $p$-value $>0.05$ at the Kruskal-Wallis test performed between the groups). Therefore, considering homogeneous the two groups respect to the extracted radiomics metrics, RAS mutational was not considered for the following analysis.

There were no differences between the extracted radiomics metrics on VIBE_FA10 and on VIBE_FA30 ( $p$-value $>0.05$ at the Kruskal-Wallis test).

Among the significant features to differentiate the front of tumor growth on VIBE_FA10, 8 textural parameters obtained an accuracy $\geq 75 \%$. Among these 8 features, the best predictor to discriminate expansive versus infiltrative front of tumor growth was HHL_glcm MaximumProbability with an accuracy of $81 \%$, a sensitivity of $92 \%$, a specificity of $62 \%$, a PPV and a NPV of $80 \%$ and $82 \%$, respectively.

Among the significant features to differentiate the front of tumor growth on VIBE_FA30, 15 textural parameters obtained an accuracy $\geq 75 \%$. Among these 15 features, the best predictor to discriminate expansive versus infiltrative front of tumor growth was HLH_glcm MaximumProbability (the same feature of previous case obtained with another wavelet filter HLH respect to HHL) with an accuracy of $84 \%$, a sensitivity of $83 \%$, a specificity of $82 \%$, a PPV and a NPV of $89 \%$ and $74 \%$, respectively. Significant radiomics metrics for each outcome at univariate analysis are reported in Table 3. 
Table 3. Significant radiomics features for each considered outcome.

\begin{tabular}{|c|c|c|c|c|c|c|c|c|}
\hline \multirow{2}{*}{$\begin{array}{c}\text { Significant Textural } \\
\text { Features } \\
\text { Extracted by }\end{array}$} & $\begin{array}{l}\text { VIBE_FA10 Respect } \\
\text { to the Front of Tumor } \\
\text { Growth }\end{array}$ & $\begin{array}{l}\text { VIBE_FA30 Respect } \\
\text { to the Front of Tumor } \\
\text { Growth }\end{array}$ & $\begin{array}{l}\text { VIBE_FA10 Respect } \\
\text { to the Tumor } \\
\text { Budding }\end{array}$ & $\begin{array}{l}\text { VIBE_FA30 Respect } \\
\text { to the Tumor } \\
\text { Budding }\end{array}$ & $\begin{array}{c}\text { VIBE_FA10 Respect } \\
\text { to the Mucinous } \\
\text { Type }\end{array}$ & $\begin{array}{c}\text { VIBE_FA30 Respect } \\
\text { to the Mucinous } \\
\text { Type }\end{array}$ & $\begin{array}{l}\text { VIBE_FA10 Respect } \\
\text { to Recurrence }\end{array}$ & $\begin{array}{l}\text { VIBE_FA30 } \\
\text { Respect to } \\
\text { Recurrence }\end{array}$ \\
\hline & $\begin{array}{c}\text { Wavelet__ } \\
\text { HHL_glcm } \\
\text { MaximumProbability }\end{array}$ & $\begin{array}{c}\text { Wavelet__ } \\
\text { HLH_glcm } \\
\text { MaximumProbability }\end{array}$ & $\begin{array}{c}\text { Wavelet_- } \\
\text { HHL_glcm_- } \\
\text { MaximumProbability }\end{array}$ & $\begin{array}{l}\text { Original_glcm_- } \\
\text { InverseVariance }\end{array}$ & $\begin{array}{l}\text { Wavelet__ } \\
\text { HHH_ngtdm } \\
\text { Busyness }\end{array}$ & $\begin{array}{c}\text { Wavelet__ } \\
\text { HHL_glszm_- } \\
\text { ZoneVariance }\end{array}$ & $\begin{array}{c}\text { Wavelet__ } \\
\text { LLH_glrlm_- } \\
\text { ShortRunEmphasis }\end{array}$ & $\begin{array}{l}\text { Wavelet_ } \\
\text { LHL_glcm } \\
\text { Correlation }\end{array}$ \\
\hline AUC & 0.66 & 0.68 & 0.70 & 0.70 & 0.65 & 0.63 & 0.48 & 0.74 \\
\hline Sensitivity & 0.92 & 0.83 & 0.94 & 0.96 & 0.42 & 0.46 & 0.31 & 0.52 \\
\hline Specificity & 0.62 & 0.82 & 0.68 & 0.65 & 0.95 & 0.96 & 1.00 & 0.97 \\
\hline NPV & 0.82 & 0.74 & 0.81 & 0.83 & 0.86 & 0.87 & 0.84 & 0.85 \\
\hline Accuracy & 0.81 & 0.84 & 0.88 & 0.89 & 0.84 & 0.85 & 0.85 & 0.86 \\
\hline Cut-off & 0.28 & 0.28 & 0.28 & 0.35 & 1306.26 & $1,289,504.66$ & 0.84 & 0.46 \\
\hline
\end{tabular}

Note: GLCM, Gray Level Co-occurrence Matrix; GLSZM, Gray Level Size Zone Matrix; GLRLM, Gray Level Run Length Matrix; GLDM, Gray Level Dependence Matrix; NGTDM, Neighboring Gray Tone Difference Matrix. 
Among the significant features to differentiate the tumor budding on VIBE_FA10, 8 textural parameters obtained an accuracy $\geq 85 \%$. Among these 8 features, the best predictor to discriminate tumor budding was again the HHL_glcm_MaximumProbability with an accuracy of $88 \%$, a sensitivity of $94 \%$, a specificity of $68 \%$, a PPV and a NPV of $89 \%$ and $81 \%$, respectively.

Among the significant features to differentiate the tumor budding on VIBE_FA30, 16 textural parameters obtained an accuracy $\geq 85 \%$. Among these 16 features, the best predictor to discriminate tumor budding was Inverse Variance obtained by the original GLCM matrix with an accuracy of $89 \%$, a sensitivity of $96 \%$, a specificity of $65 \%$, a PPV and a NPV of $89 \%$ and $83 \%$, respectively.

Among the significant features to differentiate the mucinous type of tumor on VIBE_FA10, 8 textural parameters obtained an accuracy $\geq 80 \%$. Among these 8 features, the best predictor to differentiate the mucinous type of tumor was the HHH_ngtdm_Busyness with an accuracy of $84 \%$, a sensitivity of $65 \%$, a specificity of $42 \%$, a PPV and a NPV of $69 \%$ and $86 \%$, respectively.

Among the significant features to differentiate the mucinous type of tumor on VIBE_FA30, 12 textural parameters obtained an accuracy $\geq 80 \%$. Among these 12 features, the best predictor to differentiate the mucinous type of tumor was the HHL_glszm_ZoneVariance with an accuracy of $85 \%$, a sensitivity of $46 \%$, a specificity of $95 \%$, a PPV and a NPV of $71 \%$ and $87 \%$, respectively.

Among the significant features to identify tumor recurrence on VIBE_FA10, 3 textural parameters obtained an accuracy $\geq 80 \%$. Among these 3 features, the best predictor to identify tumor recurrence was the LLH_glrlm_ShortRunEmphasis with accuracy of 85\%, a sensitivity of $31 \%$, a specificity of $100 \%$, a PPV and a NPV of $100 \%$ and $84 \%$, respectively.

Among the significant features to identify tumor recurrence on VIBE_FA30, 8 textural parameters obtained an accuracy $\geq 80 \%$. Among these 8 features, the best predictor to identify tumor recurrence was the LHL_glcm_Correlation with an accuracy of $86 \%$, a sensitivity of $52 \%$, a specificity of $97 \%$, a PPV and a NPV of $84 \%$ and $85 \%$, respectively.

In total, 26 features extracted by VIBE_FA10 were resulted significant at univariate analysis while 48 were resulted significand among those extracted on VIBE_FA30. Figure 2 shows a heat map.

\subsection{Multivariate Analysis Findings}

\subsubsection{Linear Regression Analysis Findings}

Linear regression models obtained good results in each considered classification problem (1. Front of tumor growth: expansive versus infiltrative; 2 . tumor budding: high grade versus low grade or absent; 3. mucinous type; 4 . presence of recurrence) with an accuracy in the range of 72 to $89 \%$ Tables 4 and 5, Figures 3 and 4 . The best linear regression model was obtained in the identification of the front of tumor growth considering the height textural significant metrics by VIBE_FA10 (AUC of 72\%, an accuracy of $89 \%$; sensitivity of $93 \%$ and a specificity of $82 \%$ ). The coefficients of this linear models are reported in the Table 6. 
Heat map of 26 significant features extracted by VIBE_FA10

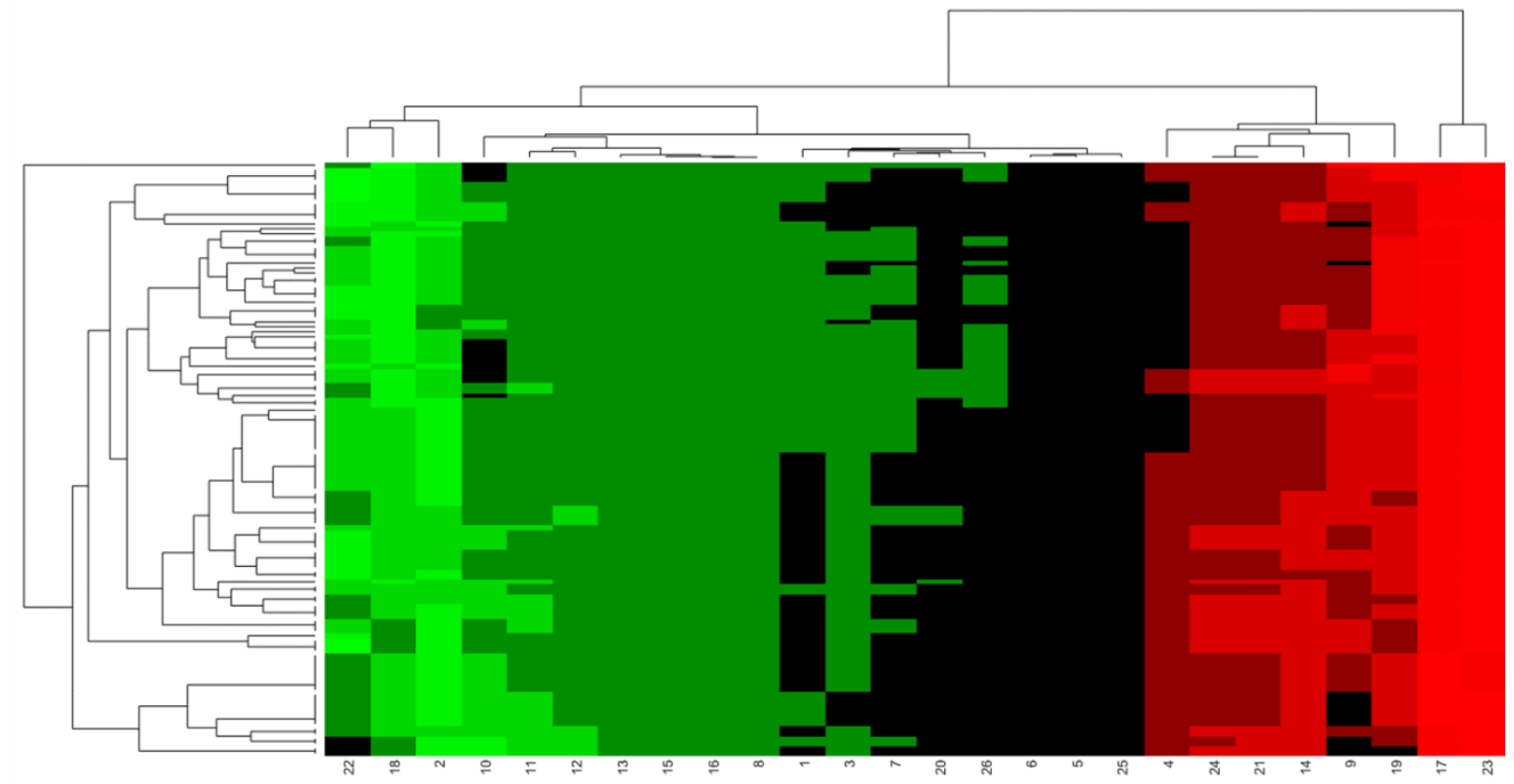

Heat map of 48 significant features extracted by VIBE_FA30

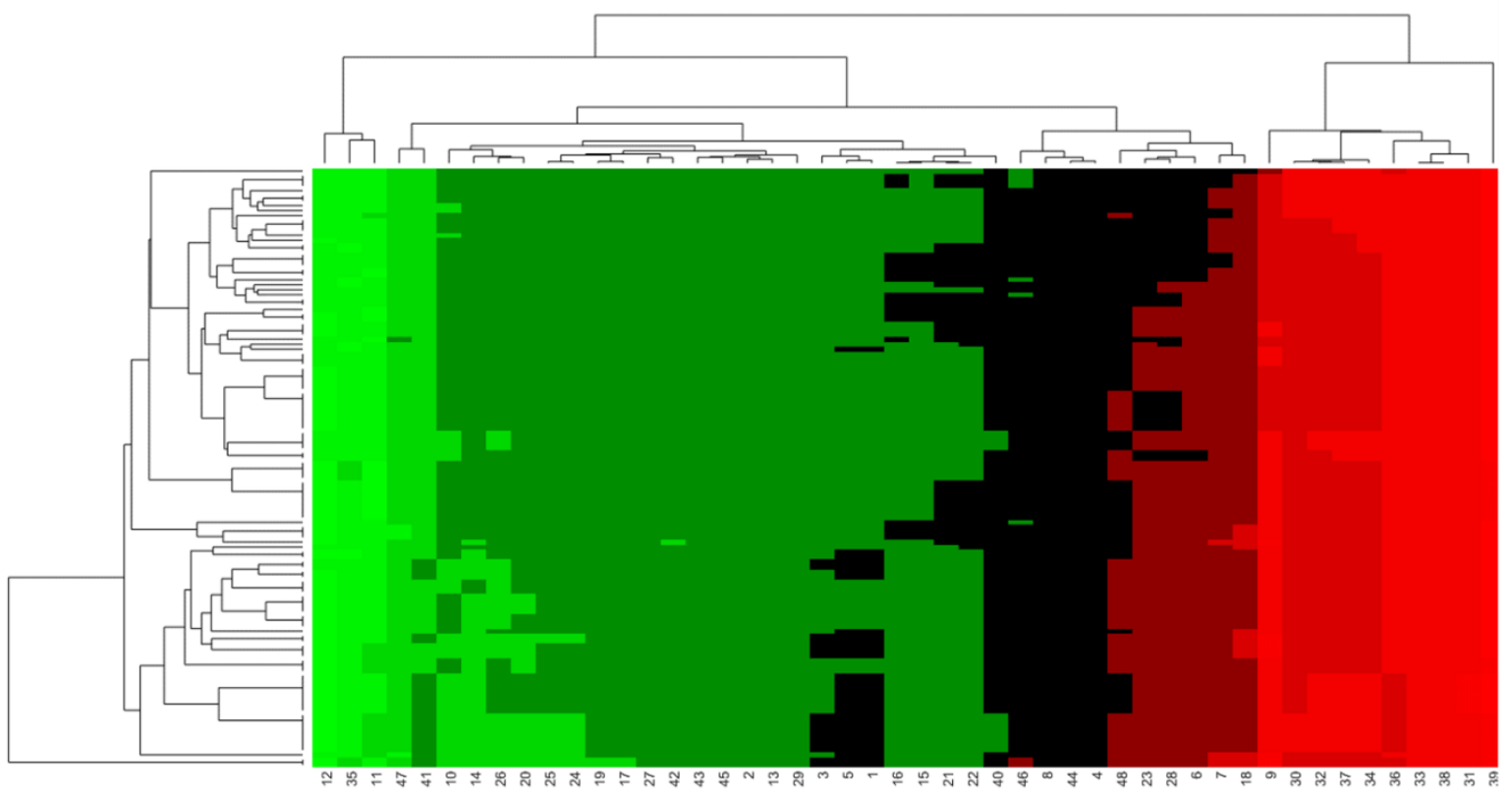

Figure 2. Heat maps pf significant radiomics features (26 extracted by VIBE_FA10 and 48 extracted by VIBE_FA30). 
Table 4. Linear regression and Pattern recognition analysis with significant features from the VIBE_FA10.

\begin{tabular}{|c|c|c|c|c|c|c|c|}
\hline $\begin{array}{c}\text { Linear Regression of Significant } \\
\text { Features }\end{array}$ & AUC & Sensitivity & Specificity & PPV & NPV & Accuracy & Cut-Off \\
\hline $\begin{array}{l}\text { Linear regression of the textural } \\
\text { features extracted from the } \\
\text { VIBE_FA10 with respect to the } \\
\text { front of tumor growth }\end{array}$ & 0.72 & 0.93 & 0.82 & 0.90 & 0.88 & 0.89 & 1.49 \\
\hline $\begin{array}{l}\text { Linear regression of the textural } \\
\text { features extracted from the } \\
\text { VIBE_FA10 with respect to the } \\
\text { tumor budding }\end{array}$ & 0.78 & 0.84 & 0.84 & 0.94 & 0.65 & 0.84 & 1.54 \\
\hline $\begin{array}{l}\text { Linear regression of the textural } \\
\text { features extracted from the } \\
\text { VIBE_FA10 with respect to the } \\
\text { mucinous type }\end{array}$ & 0.80 & 0.85 & 0.82 & 0.56 & 0.95 & 0.83 & 0.28 \\
\hline $\begin{array}{l}\text { Linear regression of the textural } \\
\text { features extracted from the } \\
\text { VIBE_FA10 with respect to the } \\
\text { recurrence presence }\end{array}$ & 0.63 & 0.52 & 0.88 & 0.59 & 0.84 & 0.79 & 3.81 \\
\hline $\begin{array}{l}\text { Pattern Recognition Analysis } \\
\text { with Significant Features }\end{array}$ & Dataset & AUC & Accuracy & Sensitivity & Specificity & $\begin{array}{l}\text { Training } \\
\text { Time [sec] }\end{array}$ & $\begin{array}{l}\text { Model Type and } \\
\text { Parameters }\end{array}$ \\
\hline \multirow{8}{*}{$\mathrm{KNN}$} & Training set & 0.96 & 0.91 & 0.84 & 0.95 & 8.7 & \multirow{8}{*}{$\begin{array}{l}\text { Weighted KNN; } \\
\text { number of } \\
\text { neighbors:10; } \\
\text { distance metric: } \\
\text { Euclidean; } \\
\text { distance weight: } \\
\text { squared inverse }\end{array}$} \\
\hline & $\begin{array}{l}\text { Validation } \\
\text { set }\end{array}$ & 0.97 & 0.92 & 1 & 0.86 & & \\
\hline & Training set & 0.89 & 0.93 & 0.81 & 0.97 & 3.9 & \\
\hline & $\begin{array}{l}\text { Validation } \\
\text { set }\end{array}$ & 0.9 & 0.93 & 0.73 & 1 & & \\
\hline & Training set & 0.93 & 0.89 & 0.94 & 0.73 & 3.2 & \\
\hline & $\begin{array}{l}\text { Validation } \\
\text { set }\end{array}$ & 0.95 & 0.88 & 0.91 & 0.8 & & \\
\hline & Training set & 0.91 & 0.93 & 0.99 & 0.77 & 9.21 & \\
\hline & $\begin{array}{l}\text { Validation } \\
\text { set }\end{array}$ & 0.97 & 0.94 & 0.9 & 0.91 & & \\
\hline
\end{tabular}

Table 5. Linear regression and Pattern recognition analysis with significant features from the VIBE_FA30.

\begin{tabular}{|c|c|c|c|c|c|c|c|}
\hline $\begin{array}{l}\text { Linear Regression of } \\
\text { Significant Features }\end{array}$ & AUC & Sensitivity & Specificity & PPV & NPV & Accuracy & Cut-Off \\
\hline $\begin{array}{l}\text { Linear regression of the textural } \\
\text { features extracted from the } \\
\text { VIBE_FA30 with respect to the } \\
\text { front of tumor growth }\end{array}$ & 0.55 & 0.88 & 0.56 & 0.77 & 0.74 & 0.76 & 8.81 \\
\hline $\begin{array}{l}\text { Linear regression of the textural } \\
\text { features extracted from the } \\
\text { VIBE_FA30 with respect to the } \\
\text { tumor budding }\end{array}$ & 0.65 & 0.96 & 0.64 & 0.82 & 0.91 & 0.84 & 0.56 \\
\hline $\begin{array}{l}\text { Linear regression of the textural } \\
\text { features extracted from the } \\
\text { VIBE_FA30 with respect to the } \\
\text { mucinous type }\end{array}$ & 0.26 & 1.00 & 0.04 & 0.64 & 1.00 & 0.64 & -0.17 \\
\hline $\begin{array}{l}\text { Linear regression of the textural } \\
\text { features extracted from the } \\
\text { VIBE_FA30 with respect to the } \\
\text { recurrence presence }\end{array}$ & 0.79 & 0.90 & 0.66 & 0.47 & 0.95 & 0.72 & 0.27 \\
\hline
\end{tabular}


Table 5. Cont.

\begin{tabular}{|c|c|c|c|c|c|c|c|}
\hline $\begin{array}{c}\text { Pattern Recognition } \\
\text { Analysis with Significant } \\
\text { Features }\end{array}$ & Dataset & AUC & Accuracy & Sensitivity & Specificity & $\begin{array}{l}\text { Training } \\
\text { time [sec] }\end{array}$ & $\begin{array}{l}\text { Model Type } \\
\text { and } \\
\text { Parameters }\end{array}$ \\
\hline \multirow{8}{*}{ KNN } & Training set & 0.96 & 0.90 & 0.91 & 0.89 & 13.4 & \multirow{8}{*}{$\begin{array}{c}\text { Weighted } \\
\text { KNN; number } \\
\text { of neighbors:10; } \\
\text { distance metric: } \\
\text { Euclidean; } \\
\text { distance } \\
\text { weight: } \\
\text { squared } \\
\text { inverse }\end{array}$} \\
\hline & $\begin{array}{c}\text { Validation } \\
\text { set }\end{array}$ & 0.95 & 0.80 & 0.67 & 1 & & \\
\hline & Training set & 0.94 & 0.93 & 0.84 & 0.96 & 8.3 & \\
\hline & $\begin{array}{c}\text { Validation } \\
\text { set }\end{array}$ & 0.94 & 0.89 & 0.89 & 0.89 & & \\
\hline & Training set & 0.93 & 0.91 & 0.96 & 0.73 & 7.51 & \\
\hline & $\begin{array}{c}\text { Validation } \\
\text { set }\end{array}$ & 0.89 & 0.88 & 0.89 & 0.8 & & \\
\hline & Training set & 0.9 & 0.94 & 0.98 & 0.84 & 8.4 & \\
\hline & $\begin{array}{l}\text { Validation } \\
\text { set }\end{array}$ & 0.85 & 0.91 & 0.94 & 0.8 & & \\
\hline
\end{tabular}
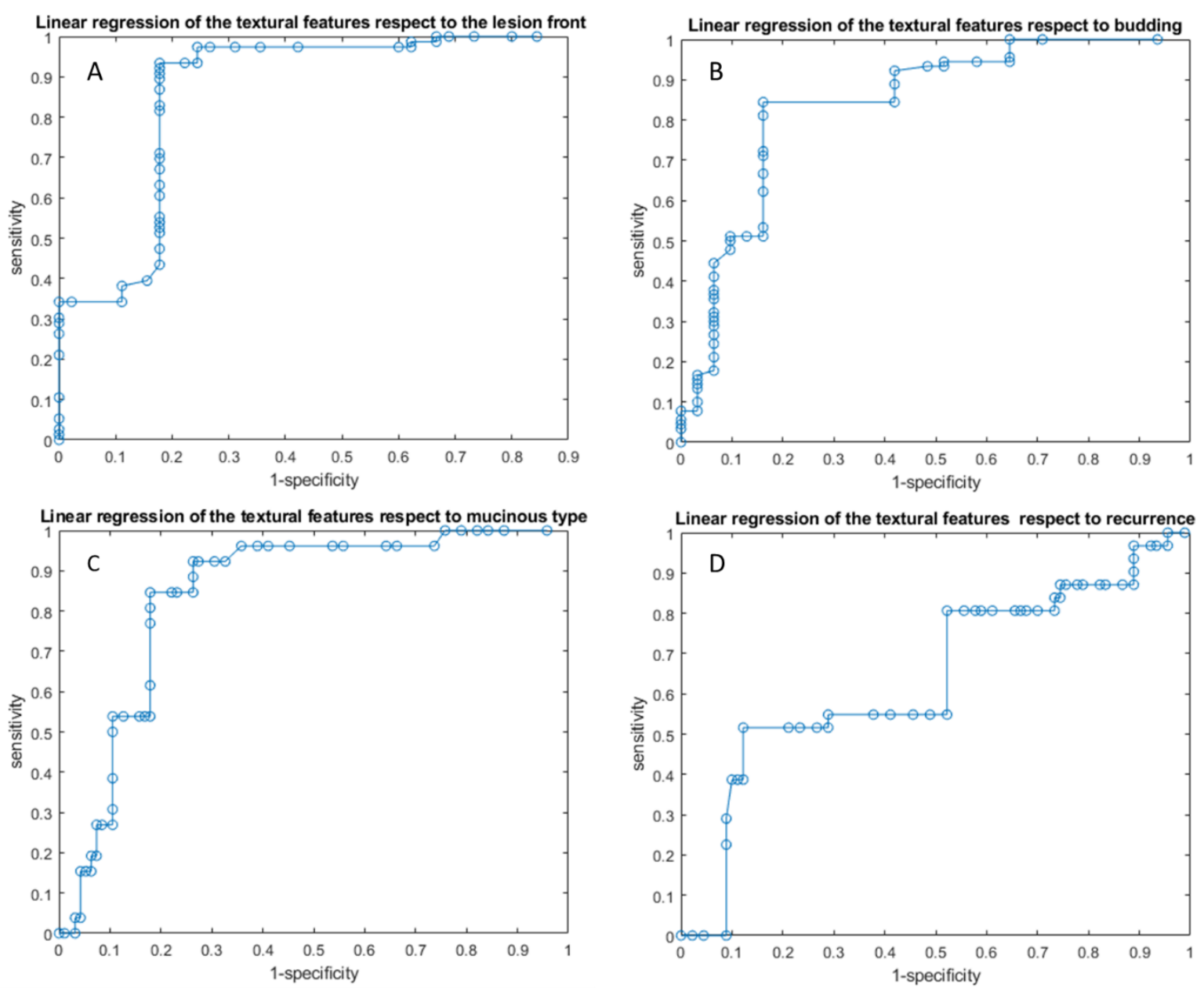

Figure 3. ROC curves of linear regression analysis respect to the front of tumor growth (A), the tumor budding (B), the tumor mucinous type (C), recurrence presence (D) obtained considering significant features extracted by VIBE_FA10. 

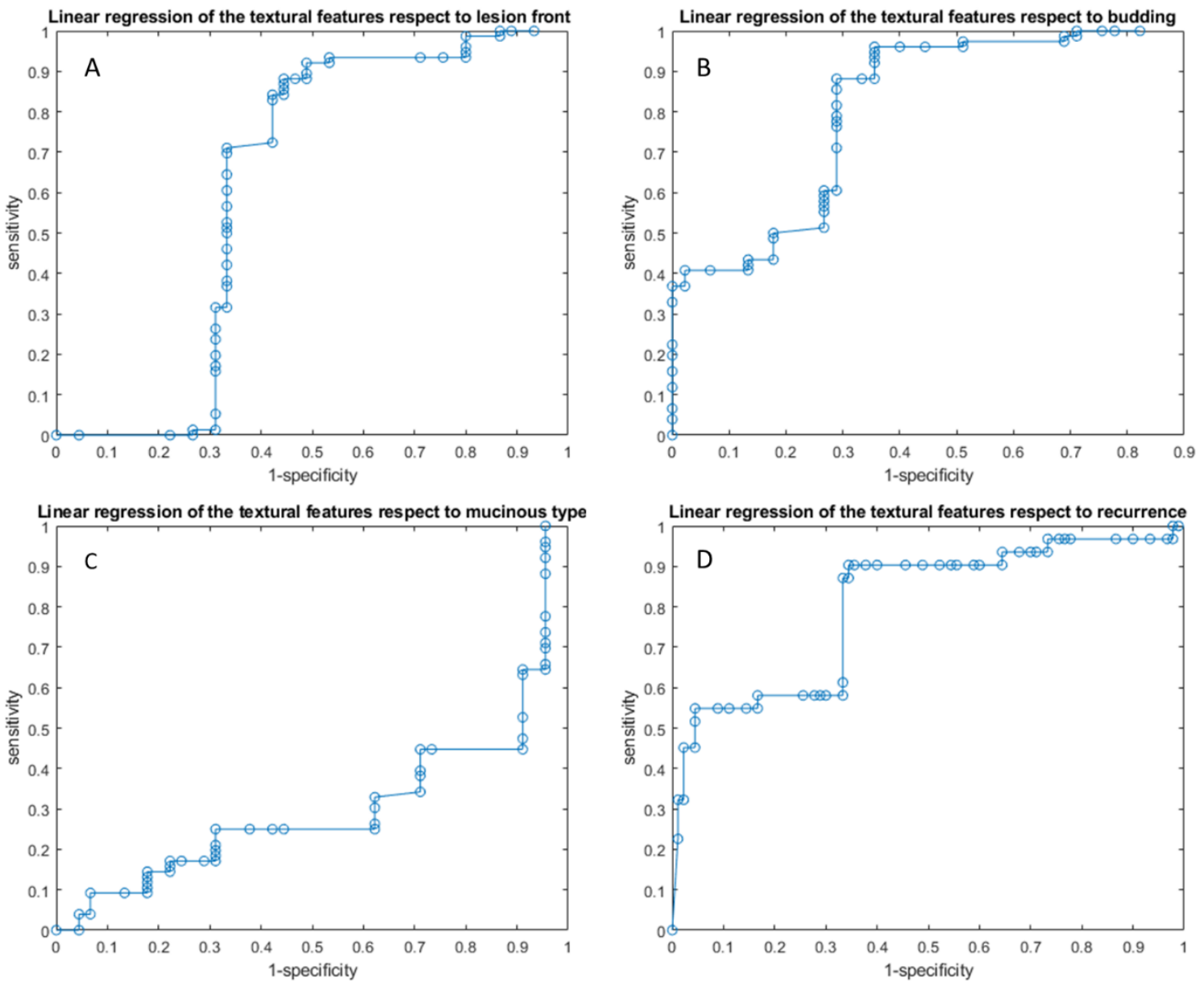

Figure 4. ROC curves of linear regression analysis respect to the front of tumor growth (A), the tumor budding (B), the tumor mucinous type (C), recurrence presence (D) obtained considering significant features extracted by VIBE_FA30.

Table 6. Linear regression model parameters.

\begin{tabular}{ccc}
\hline $\begin{array}{c}\text { Linear Regression of the Textural Features Extracted by } \\
\text { VIBE_FA10 with Respect to the Front of Tumor Growth }\end{array}$ & Coefficients & $p$ Value \\
\hline Intercept & -7.37 & 0.08 \\
\hline original_shape_SurfaceVolumeRatio & -0.85 & 0.58 \\
\hline wavelet_LHL_glszm_SmallAreaLowGrayLevelEmphasis & 1.50 & 0.19 \\
\hline wavelet_LLH_glcm_InverseVariance & 4.15 & 0.00 \\
\hline wavelet_HHH_glrlm_ShortRunHighGrayLevelEmphasis & 0.14 & 0.00 \\
\hline wavelet_HHH_glrlm_ShortRunEmphasis & 6.25 & 0.51 \\
\hline wavelet_HHH_glrlm_RunPercentage & -6.73 & 0.57 \\
\hline wavelet_HHH_glrlm_RunLengthNonUniformityNormalized & 5.15 & 0.40 \\
\hline wavelet_HHL_glcm_MaximumProbability & 16.11 & 0.00 \\
\hline
\end{tabular}


Table 6. Cont.

\begin{tabular}{|c|c|c|c|}
\hline $\begin{array}{l}\text { Linear Regression of the Textural Features } \\
\text { ExtractedbyVIBE_FA10 with Respect to the Tumor Budding }\end{array}$ & Coefficients & $p$ value & $p$ value \\
\hline Intercept & -17.88 & 0.00 & \multirow{9}{*}{0.000} \\
\hline wavelet_HLL_gldm_LargeDependenceLowGrayLevelEmphasis & 0.11 & 0.32 & \\
\hline wavelet_HLL_glrlm_LongRunLowGrayLevelEmphasis & -3.28 & 0.33 & \\
\hline wavelet_HLL_glszm_GrayLevelNonUniformityNormalized & -4.28 & 0.05 & \\
\hline wavelet_LLH_glrlm_GrayLevelNonUniformityNormalized & 2.15 & 0.07 & \\
\hline wavelet_HLH_glcm_JointEnergy & 48.41 & 0.00 & \\
\hline wavelet_HLH_firstorder_10Percentile & 0.00 & 0.96 & \\
\hline wavelet_HHH_glcm_MaximumProbability & 1.42 & 0.87 & \\
\hline wavelet_HHL_glcm_MaximumProbability & 24.94 & 0.00 & \\
\hline $\begin{array}{l}\text { Linear Regression of the Textural Features } \\
\text { ExtractedbyVIBE_FA10 with Respect to the Mucinous Type }\end{array}$ & Coefficients & $p$ value & $p$ value \\
\hline Intercept & 3.31 & 0.14 & \multirow{9}{*}{0.000} \\
\hline wavelet_LHL_gldm_DependenceNonUniformity & 0.00 & 0.03 & \\
\hline wavelet_LHL_ngtdm_Strength & -1.20 & 0.08 & \\
\hline wavelet_LHL_ngtdm_Busyness & 0.00 & 0.07 & \\
\hline wavelet_LHH_glcm_ClusterTendency & 7.50 & 0.00 & \\
\hline wavelet_HLH_gldm_DependenceEntropy & -0.03 & 0.97 & \\
\hline wavelet_HLH_firstorder_Mean & 0.12 & 0.59 & \\
\hline wavelet_HHH_ngtdm_Busyness & 0.00 & 0.08 & \\
\hline wavelet_HHL_gldm_DependenceEntropy & -1.44 & 0.04 & \\
\hline $\begin{array}{l}\text { Linear Regression of the Textural Features } \\
\text { ExtractedbyVIBE_FA10 with Respect to the Recurrence Presence }\end{array}$ & Coefficients & $p$ value & $p$ value \\
\hline Intercept & -2.95 & 0.05 & \multirow{4}{*}{0.030} \\
\hline wavelet_LLH_glrlm_ShortRunEmphasis & 8.19 & 0.04 & \\
\hline wavelet_LLH_glrlm_RunLengthNonUniformityNormalized & -5.25 & 0.10 & \\
\hline wavelet_HHH_ngtdm_Busyness & 0.00 & 0.49 & \\
\hline $\begin{array}{c}\text { Linear Regression of the Textural Features } \\
\text { ExtractedbyVIBE_FA30 with Respect to the Front of Tumor } \\
\text { Growth }\end{array}$ & Coefficients & $p$ value & $p$ value \\
\hline Intercept & -4.868 & 0.010 & \multirow{8}{*}{0.000} \\
\hline original_glcm_InverseVariance & 1.484 & 0.260 & \\
\hline wavelet_HLL_gldm_GrayLevelVariance & -4.907 & 0.206 & \\
\hline wavelet_HLL_glcm_InverseVariance & 4.257 & 0.165 & \\
\hline wavelet_HLL_glcm_DifferenceVariance & -0.967 & 0.182 & \\
\hline wavelet_HLL_glcm_SumEntropy & 1.329 & 0.195 & \\
\hline wavelet_HLL_glcm_SumSquares & -0.462 & 0.540 & \\
\hline wavelet_HLL_firstorder_RobustMeanAbsoluteDeviation & 0.360 & 0.244 & \\
\hline
\end{tabular}


Table 6. Cont.

\begin{tabular}{|c|c|c|c|}
\hline $\begin{array}{c}\text { Linear Regression of the Textural Features ExtractedbyVIBE_FA30 } \\
\text { with Respect to the Front of Tumor Growth }\end{array}$ & Coefficients & $p$ value & $p$ value \\
\hline wavelet_HLL_firstorder_MeanAbsoluteDeviation & -0.612 & 0.161 & \\
\hline wavelet_HLL_firstorder_RootMeanSquared & 0.153 & 0.273 & \\
\hline wavelet_HLL_firstorder_RootMeanSquared & 0.823 & 0.717 & \\
\hline wavelet_HLL_firstorder_Variance & 0.010 & 0.162 & \\
\hline wavelet_LHH_glrlm_ShortRunLowGrayLevelEmphasis & -0.018 & 0.944 & \\
\hline wavelet_HLH_glcm_MaximumProbability & 8.613 & 0.051 & \\
\hline wavelet_HLH_glcm_MaximumProbability & 0.903 & 0.817 & \\
\hline wavelet_LLL_gldm_SmallDependenceLowGrayLevelEmphasis & 24.740 & 0.440 & \\
\hline $\begin{array}{c}\text { Linear Regression of the Textural Features ExtractedbyVIBE_FA30 } \\
\text { with Respect to the Tumor Budding }\end{array}$ & Coefficients & $p$ value & $p$ value \\
\hline Intercept & 5.220 & 0.521 & \multirow{17}{*}{0.000} \\
\hline original_glcm_InverseVariance & 2.978 & 0.008 & \\
\hline wavelet_HLL_glcm_JointEnergy & -12.594 & 0.008 & \\
\hline wavelet_HLL_glcm_Idm & 114.999 & 0.000 & \\
\hline wavelet_HLL_glcm_Id & -123.518 & 0.002 & \\
\hline wavelet_HLL_firstorder_Uniformity & -18.651 & 0.011 & \\
\hline wavelet_HLL_firstorder_10Percentile & -0.005 & 0.801 & \\
\hline wavelet_HLL_glrlm_GrayLevelNonUniformityNormalized & 14.712 & 0.009 & \\
\hline wavelet_HLL_glszm_GrayLevelNonUniformityNormalized & -0.804 & 0.458 & \\
\hline wavelet_LHL_glcm_Idm & -71.494 & 0.012 & \\
\hline wavelet_LHL_glcm_Id & 79.815 & 0.014 & \\
\hline wavelet_LHH_firstorder_10Percentile & -0.003 & 0.941 & \\
\hline wavelet_LLH_firstorder_Uniformity & 10.540 & 0.002 & \\
\hline wavelet_LLH_glrlm_GrayLevelNonUniformityNormalized & -12.808 & 0.002 & \\
\hline wavelet_LLH_glszm_GrayLevelNonUniformityNormalized & 2.066 & 0.156 & \\
\hline wavelet_HHL_glcm_JointEnergy & 1.672 & 0.853 & \\
\hline wavelet_HHL_firstorder_10Percentile & 0.202 & 0.006 & \\
\hline $\begin{array}{c}\text { Linear Regression of the Textural Features ExtractedbyVIBE_FA30 } \\
\text { with Respect to the Mucinous Type }\end{array}$ & Coefficients & $p$ value & $p$ value \\
\hline Intercept & 13.293 & 0.018 & \multirow{9}{*}{0.000} \\
\hline original_shape_SurfaceVolumeRatio & -2.669 & 0.000 & \\
\hline wavelet_LHH_gldm_DependenceNonUniformity & 0.004 & 0.113 & \\
\hline wavelet_LHH_gldm_GrayLevelNonUniformity & 0.000 & 0.710 & \\
\hline wavelet_HLH_gldm_DependenceNonUniformity & -0.014 & 0.000 & \\
\hline wavelet_HLH_glrlm_GrayLevelNonUniformity & 0.005 & 0.003 & \\
\hline wavelet_HHH_gldm_DependenceNonUniformity & -0.002 & 0.473 & \\
\hline wavelet_HHH_glszm_ZonePercentage & 67.121 & 0.000 & \\
\hline wavelet_HHH_ngtdm_Busyness & 0.000 & 0.000 & \\
\hline
\end{tabular}


Table 6. Cont.

\begin{tabular}{ccc}
\hline $\begin{array}{c}\text { Linear Regression of the Textural Features } \\
\text { ExtractedbyVIBE_FA30 with Respect to the Mucinous Type }\end{array}$ & Coefficients & $p$ value \\
\hline wavelet_HHL_gldm_DependenceNonUniformity & 0.012 & 0.000 \\
\hline wavelet_HHL_glrlm_GrayLevelNonUniformity & -0.005 & 0.004 \\
\hline wavelet_HHL_glszm_ZoneVariance & 0.000 & 0.462 \\
\hline wavelet_LLL_glcm_Idmn & -12.578 & 0.025 \\
\hline Einear Regression of the Textural Features & Coefficients & value \\
\hline Intercept & -0.018 & 0.966 \\
\hline original_glszm_ZonePercentage & 0.852 & 0.540 \\
\hline wavelet_HLL_glcm_Correlation & -0.464 & 0.684 \\
\hline wavelet_LHL_glcm_Correlation & 5.324 & 0.001 \\
\hline wavelet_LHL_glcm_SumEntropy & -0.243 & 0.474 \\
\hline wavelet_LHL_glcm_Imc2 & v2.956 & 0.037 \\
\hline wavelet_LHL_glcm_ClusterTendency & -0.014 & 0.891 \\
\hline wavelet_HHL_glrlm_HighGrayLevelRunEmphasis & 2.266 & 0.033 \\
\hline
\end{tabular}

\subsubsection{Pattern Recognition Approaches Findings}

Considering the significant texture metrics tested with pattern recognition approaches, the best performance for each outcome (1. Front of tumor growth: expansive versus infiltrative; 2 . tumor budding: high grade versus low grade or absent; 3 . mucinous type and 4. presence of recurrence) was reached by a $\mathrm{KNN}$, both considering the features extracted by VIBE_FA10 and VIBE_FA30. The accuracy was always major to $88 \%$ (Tables 4 and 5, Figures 5 and 6 ) both on training and validation set and the best results was obtained in the identification of recurrence with the 3 textural significant features extracted by VIBE_FA10 (AUC of $91 \%$, an accuracy of $93 \%$; sensitivity of $99 \%$ and a specificity of $77 \%$ ). 

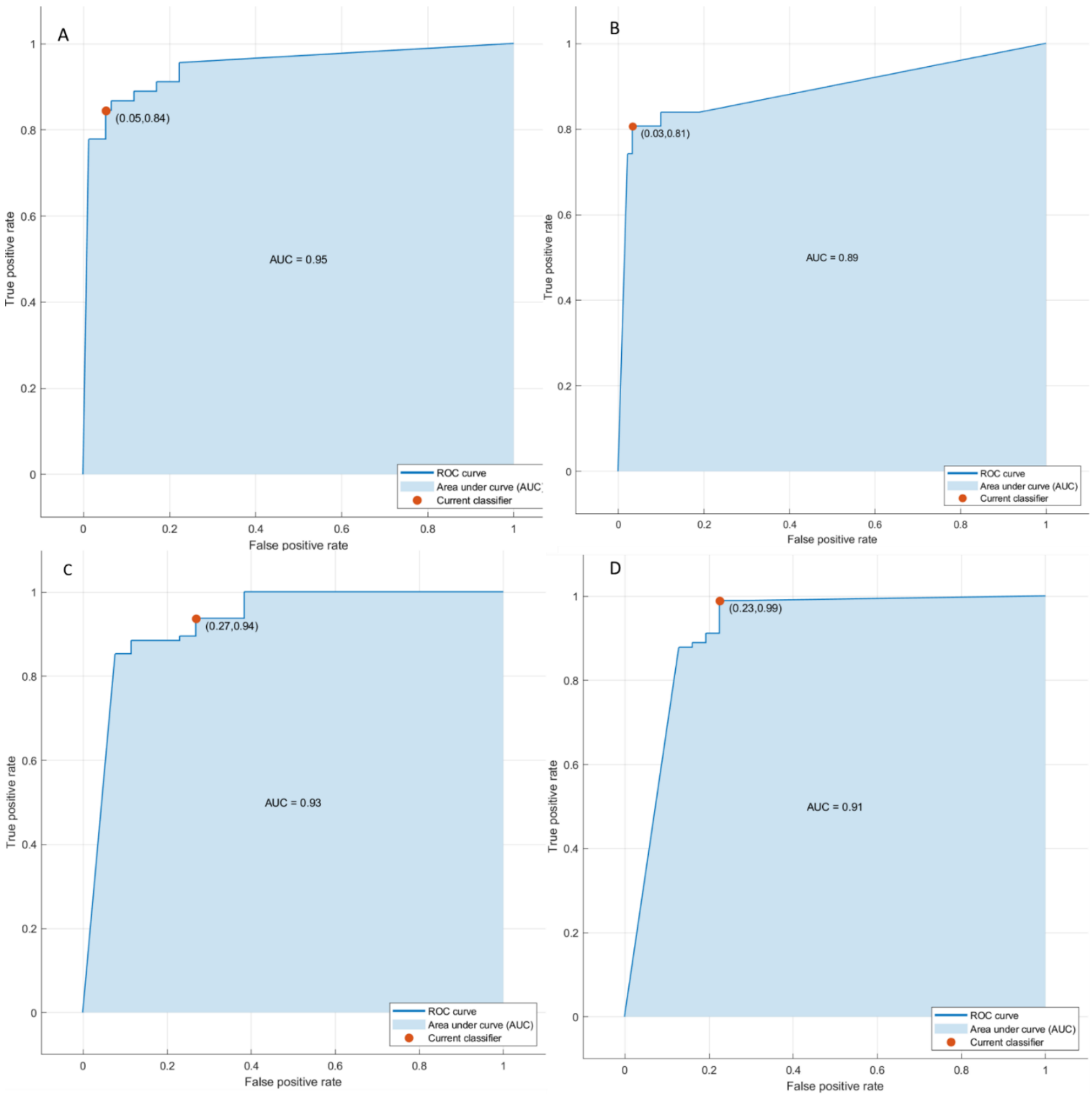

Figure 5. ROC curves of KNN respect to the front of tumor growth (A), the tumor budding (B), the tumor mucinous type (C), recurrence presence (D) obtained considering significant features extracted by VIBE_FA10. 

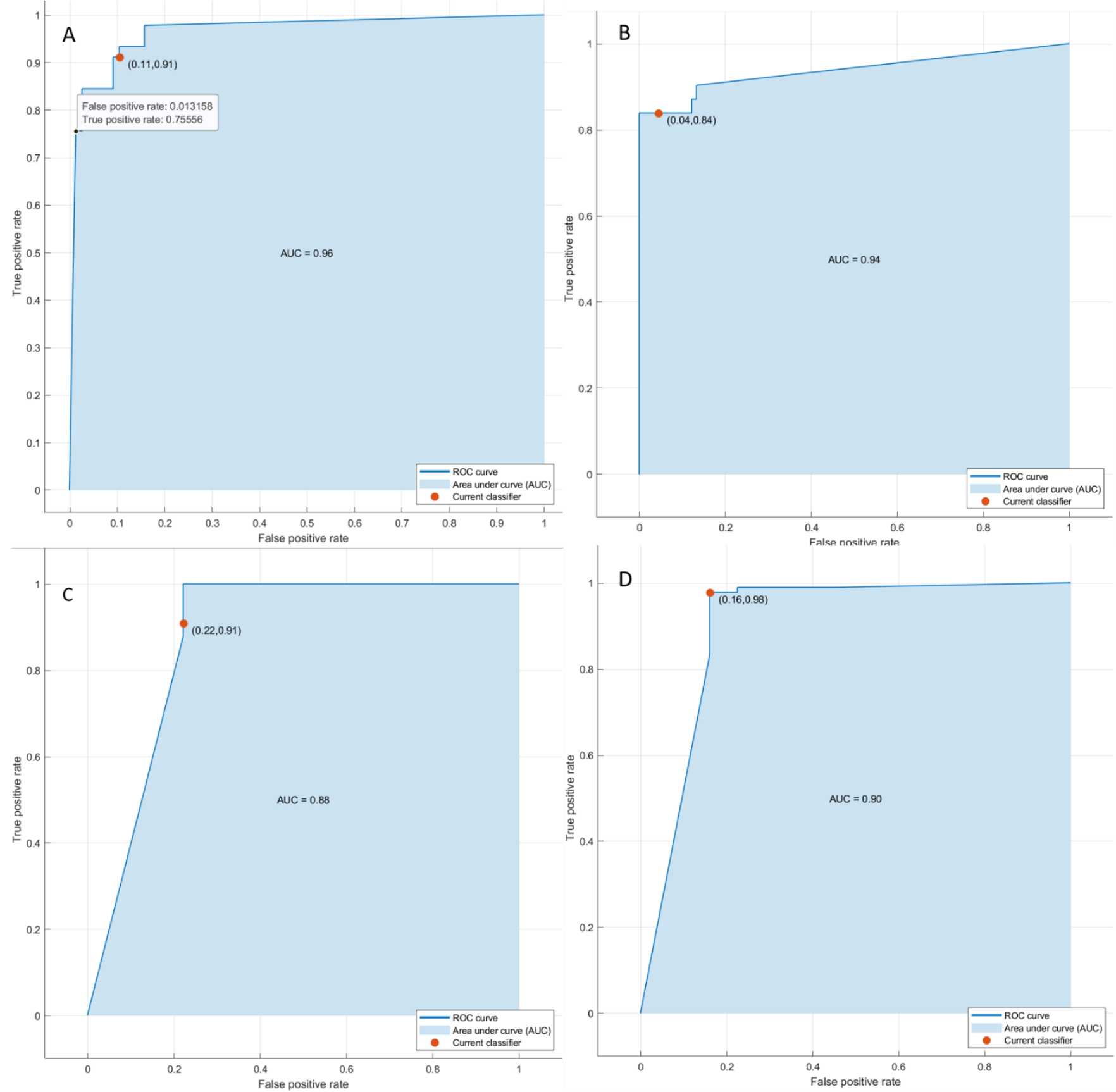

Figure 6. ROC curves of KNN respect to the front of tumor growth (A), the tumor budding (B), the tumor mucinous type $(C)$, recurrence presence $(D)$ obtained considering significant features extracted by VIBE_FA30.

\section{Discussions}

Ours results confirmed the capacity of radiomics to identify as biomarkers, several prognostic features that could affect the treatment choice in patients with liver metastases, in order to obtain a more personalized approach. In fact, the possibility to correlate radiomics parameters to RAS status offers notable advantages over qualitative imaging assessment, allowing one to tailor cancer therapy to the patient, to predict response to treatment, to distinguish favorable subsets of patients from those with poor prognosis, to select patients that may benefit of surgical treatment. Literature data underlines the role of several features, as RAS mutation, front of tumor growth, tumor budding and mucinous type as a strong prognostic and predictive biomarker in patients subjected to target therapy or surgical 
resection. In this scenario, our results confirmed the possibility of radiomics to allow one to tailor cancer therapy at the patient, to predict response to treatment, to detect favorable subsets of patients from those with poor prognosis and to select patients that may benefit from surgical treatment $[5,6]$.

Our results were confirmed by external validation dataset. We obtained a good performance considering the single textural significant metric in the identification of front of tumor growth (expansive versus infiltrative) and tumor budding (high grade versus low grade or absent), in the recognition of mucinous type and in the detection of recurrences. The median value of intraclass correlation coefficients for features was 0.92 .

With regard to the front of tumor growth on VIBE_FA10, the best performance was obtained with HHL_glcm_MaximumProbability with an accuracy of $81 \%$; while on VIBE_FA30, the best performance was with HLH_glcm_MaximumProbability (the same feature of previous case obtained with another wavelet filter HLH respect to HHL) with an accuracy of $84 \%$. Among significant features to differentiate the tumor budding on VIBE_FA10, the best predictot was again the HHL_glcm_MaximumProbability with an accuracy of $88 \%$ while on VIBE_FA30, the best performance was of the Inverse Variance extracted by the original GLCM matrix with an accuracy of $89 \%$.

Regarding to differentiate the mucinous type of tumor on VIBE_FA10, the best predictor was the HHH_ngtdm_Busyness with an accuracy of $84 \%$ while on VIBE_FA30, the best performance was obtained by the HHL_glszm_ZoneVariance with an accuracy of 85\%.

Among the significant features to identify tumor recurrence on VIBE_FA10, the best performance was obtained by the LLH_glrlm_ShortRunEmphasis with an accuracy of 85\% while on VIBE_FA30, the best predictor was the LHL_glcm_Correlation with an accuracy of $86 \%$.

Therefore, all of the significant and better predictors for each outcome except that the Inverse Variance obtained by the original GLCM matrix were Higher-order statistics features obtained by statistical methods after wavelet transform. However, all of these metrics capture certain statistical regularities of tumor lesions through images linked to the heterogeneity of gray levels on the segmented volume of interest.

Considering a linear regression models or neural network classifiers in a multivariate approach was possible to increase the performance in terms of accuracy, sensitivity, and specificity. The best linear regression model was obtained in the identification of the front of tumor growth considering the height textural significant metrics by VIBE_FA10 (AUC of $72 \%$, an accuracy of $89 \%$; sensitivity of $93 \%$ and a specificity of $82 \%$ ) while the best results with a KNN was obtained in the identification of recurrence with the 3 textural significant features (AUC of $91 \%$, an accuracy of $93 \%$; sensitivity of $99 \%$ and a specificity of $77 \%$ ).

Research has shown the association between entropy and prognosis [31-40]. Homogeneity in the texture of healthy liver tissue is predictive of worse survival. Andersen et al. [32] demonstrated a link between homogeneity features and overall survival (OS). Rahmim et al. [37] showed as radiomics data of heterogeneity, obtained by FDG PET study, were prognosticators of lower OS [37]. Research has shown the degree of skewness was inversely correlated with KRAS status, while the entropy was related to OS [34]. Moreover, the opportunity to identify the patients for recurrence has been shown [37-41]. Ravanelli et al. [39] correlated high CT uniformity and low OS and Progression Free Survival (PFS).

Radiomics and radiogenomics are emerging fields with important weaknesses that need to be taken into account. The main limit is the heterogeneity of software analysis and the variety of the metrics in different hospitals. Therefore, the segmentation of lesion may affect results [41].

The present study had several limitations: (1) the small population size considered, although the analysis was conducted on a homogeneous sample and on all individual lesions; (2) the retrospective nature of the study, (3) a manual segmentation, that, although research has supported automatic segmentation to avoid inter-observer variability, in our opinion, the manual approach is more realistic. Moreover, we did not assess the impact of the contrast administration and the different phases of contrast study (arterial, portal 
and transitional phase) respect to EOB-phase, data that we are evaluating in a future paper. However, we evaluated the impact of different flip angle (10 and 30). Additionally, we did not evaluate the impact of chemotherapy on our data.

\section{Conclusions}

Ours results confirmed the capacity of radiomics to identify, as biomarkers, several prognostic features that could affect the treatment choice in patients with liver metastases, in order to obtain a more personalized approach. These results were confirmed by external validation dataset. We obtained a good performance considering the single textural significant metric in the identification of front of tumor growth (expansive versus infiltrative) and tumor budding (high grade versus low grade or absent), in the recognition of mucinous type and in the detection of recurrences.

Author Contributions: Data curation, V.G.; Formal analysis, V.G. and R.F.; Investigation, V.G., F.D.M., C.C., S.V.S., F.D.A., A.O., G.N., R.G., V.P., V.M., M.C.B., F.T., F.I. and A.P.; Methodology, V.G., R.F., F.D.M., C.C., S.V.S., F.D.A., A.O., G.N., R.G., V.P., V.M., M.C.B., F.T., F.I. and A.P.; Writing-original draft, V.G.; Writing-review \& editing, V.G. All authors have read and agreed to the published version of the manuscript.

Funding: This research received no external funding.

Institutional Review Board Statement: This study aligned with National appropriate guidelines and procedures. The National Cancer Institute of Naples (80131 Naples, Italy) Ethical Committee board approved this retrospective study.

Informed Consent Statement: This study aligned with National appropriate guidelines and procedures. Renouncing the need for informed patient consent given the study nature.

Data Availability Statement: Data and material are available at https:/ / zenodo.org/record/629975 4\#.Yh-ezOjMK3A accedded on 24 February 2022.

Acknowledgments: The authors are grateful to Alessandra Trocino, librarian at the National Cancer Institute of Naples, Italy. Moreover, for the collaboration, authors are grateful for the research support to Paolo Pariate, Martina Totaro and Andrea Esposito of Radiology Division, Istituto Nazionale Tumori IRCCS Fondazione Pascale-IRCCS di Napoli, 80131 Napoli, Italy.

Conflicts of Interest: The authors declare no conflict of interest.

\section{References}

1. Granata, V.; Fusco, R.; Costa, M.; Picone, C.; Cozzi, D.; Moroni, C.; La Casella, G.V.; Montanino, A.; Monti, R.; Mazzoni, F.; et al. Preliminary Report on Computed Tomography Radiomics Features as Biomarkers to Immunotherapy Selection in Lung Adenocarcinoma Patients. Cancers 2021, 13, 3992. [CrossRef] [PubMed]

2. Granata, V.; Fusco, R.; Barretta, M.L.; Picone, C.; Avallone, A.; Belli, A.; Patrone, R.; Ferrante, M.; Cozzi, D.; Grassi, R.; et al. Radiomics in hepatic metastasis by colorectal cancer. Infect. Agent Cancer 2021, 16, 39. [CrossRef] [PubMed]

3. Fusco, R.; Piccirillo, A.; Sansone, M.; Granata, V.; Rubulotta, M.R.; Petrosino, T.; Barretta, M.L.; Vallone, P.; Di Giacomo, R.; Esposito, E.; et al. Radiomics and Artificial Intelligence Analysis with Textural Metrics Extracted by Contrast-Enhanced Mammography in the Breast Lesions Classification. Diagnostics 2021, 11, 815. [CrossRef] [PubMed]

4. Fusco, R.; Granata, V.; Mazzei, M.A.; Meglio, N.D.; Roscio, D.D.; Moroni, C.; Monti, R.; Cappabianca, C.; Picone, C.; Neri, E.; et al. Quantitative imaging decision support (QIDS ${ }^{\mathrm{TM}}$ ) tool consistency evaluation and radiomic analysis by means of 594 metrics in lung carcinoma on chest CT scan. Cancer Control 2021, 28, 1073274820985786. [CrossRef] [PubMed]

5. Granata, V.; Fusco, R.; Avallone, A.; De Stefano, A.; Ottaiano, A.; Sbordone, C.; Brunese, L.; Izzo, F.; Petrillo, A. Radiomics-Derived Data by Contrast Enhanced Magnetic Resonance in RAS Mutations Detection in Colorectal Liver Metastases. Cancers 2021, 13, 453. [CrossRef]

6. Granata, V.; Fusco, R.; Risi, C.; Ottaiano, A.; Avallone, A.; De Stefano, A.; Grimm, R.; Grassi, R.; Brunese, L.; Izzo, F.; et al. Diffusion-Weighted MRI and Diffusion Kurtosis Imaging to Detect RAS Mutation in Colorectal Liver Metastasis. Cancers 2020, 12, 2420. [CrossRef]

7. Petralia, G.; Summers, P.E.; Agostini, A.; Ambrosini, R.; Cianci, R.; Cristel, G.; Calistri, L.; Colagrande, S. Dynamic contrastenhanced MRI in oncology: How we do it. Radiol. Med. 2020, 125, 1288-1300. [CrossRef]

8. Ria, F.; Samei, E. Is regulatory compliance enough to ensure excellence in medicine? Radiol. Med. 2020, 125, 904-905. [CrossRef] 
9. Zhang, A.; Song, J.; Ma, Z.; Chen, T. Combined dynamic contrast-enhanced magnetic resonance imaging and diffusion-weighted imaging to predict neoadjuvant chemotherapy effect in FIGO stage IB2-IIA2 cervical cancers. Radiol. Med. 2020, 125, 1233-1242. [CrossRef]

10. Crimi, F.; Capelli, G.; Spolverato, G.; Bao, Q.R.; Florio, A.; Milite Rossi, S.; Cecchin, D.; Albertoni, L.; Campi, C.; Pucciarelli, S.; et al. MRI T2-weighted sequences-based texture analysis (TA) as a predictor of response to neoadjuvant chemo-radiotherapy (nCRT) in patients with locally advanced rectal cancer (LARC). Radiol. Med. 2020, 125, 1216-1224. [CrossRef]

11. Kirienko, M.; Ninatti, G.; Cozzi, L.; Voulaz, E.; Gennaro, N.; Barajon, I.; Ricci, F.; Carlo-Stella, C.; Zucali, P.; Sollini, M.; et al. Computed tomography (CT)-derived radiomic features differentiate prevascular mediastinum masses as thymic neoplasms versus lymphomas. Radiol. Med. 2020, 125, 951-960. [CrossRef] [PubMed]

12. Zhang, L.; Kang, L.; Li, G.; Zhang, X.; Ren, J.; Shi, Z.; Li, J.; Yu, S. Computed tomography-based radiomics model for discriminating the risk stratification of gastrointestinal stromal tumors. Radiol. Med. 2020, 125, 465-473. [CrossRef] [PubMed]

13. Gurgitano, M.; Angileri, S.A.; Rodà, G.M.; Liguori, A.; Pandolfi, M.; Ierardi, A.M.; Wood, B.J.; Carrafiello, G. Interventional Radiology ex-machina: Impact of Artificial Intelligence on practice. Radiol. Med. 2021, 126, 998-1006. [CrossRef]

14. Scapicchio, C.; Gabelloni, M.; Barucci, A.; Cioni, D.; Saba, L.; Neri, E. A deep look into radiomics. Radiol. Med. 2021, 126, 1296-1311. [CrossRef] [PubMed]

15. Wei, J.; Jiang, H.; Gu, D.; Niu, M.; Fu, F.; Han, Y.; Song, B.; Tian, J. Radiomics in liver diseases: Current progress and future opportunities. Liver Int. 2020, 40, 2050-2063. [CrossRef]

16. Saini, A.; Breen, I.; Pershad, Y.; Naidu, S.; Knuttinen, M.G.; Alzubaidi, S.; Sheth, R.; Albadawi, H.; Kuo, M.; Oklu, R. Radiogenomics and Radiomics in Liver Cancers. Diagnostics 2018, 9, 4. [CrossRef]

17. De la Pinta, C.; Castillo, M.E.; Collado, M.; Galindo-Pumariño, C.; Peña, C. Radiogenomics: Hunting Down Liver Metastasis in Colorectal Cancer Patients. Cancers 2021, 13, 5547. [CrossRef]

18. Nardone, V.; Reginelli, A.; Grassi, R.; Boldrini, L.; Vacca, G.; D’Ippolito, E.; Annunziata, S.; Farchione, A.; Belfiore, M.P.; Desideri, I.; et al. Delta radiomics: A systematic review. Radiol. Med. 2021, 126, 1571-1583. [CrossRef]

19. Brunese, L.; Brunese, M.C.; Carbone, M.; Ciccone, V.; Mercaldo, F.; Santone, A. Automatic PI-RADS assignment by means of formal methods. Radiol. Med. 2021, 127, 83-89. [CrossRef]

20. Van der Lubbe, M.F.J.A.; Vaidyanathan, A.; de Wit, M.; van den Burg, E.L.; Postma, A.A.; Bruintjes, T.D.; Bilderbeek-Beckers, M.A.L.; Dammeijer, P.F.M.; Bossche, S.V.; Van Rompaey, V.; et al. A non-invasive, automated diagnosis of Menière's disease using radiomics and machine learning on conventional magnetic resonance imaging: A multicentric, case-controlled feasibility study. Radiol. Med. 2021, 27, 72-82. [CrossRef]

21. Granata, V.; Fusco, R.; Avallone, A.; Cassata, A.; Palaia, R.; Delrio, P.; Grassi, R.; Tatangelo, F.; Grazzini, G.; Izzo, F.; et al. Abbreviated MRI protocol for colorectal liver metastases: How the radiologist could work in pre surgical setting. PLoS ONE 2020, 15, e241431. [CrossRef] [PubMed]

22. Granata, V.; Fusco, R.; Venanzio Setola, S.; Mattace Raso, M.; Avallone, A.; De Stefano, A.; Nasti, G.; Palaia, R.; Delrio, P.; Petrillo, A.; et al. Liver radiologic findings of chemotherapy-induced toxicity in liver colorectal metastases patients. Eur. Rev. Med. Pharmacol. Sci. 2019, 23, 9697-9706. [CrossRef] [PubMed]

23. Granata, V.; Fusco, R.; Maio, F.; Avallone, A.; Nasti, G.; Palaia, R.; Albino, V.; Grassi, R.; Izzo, F.; Petrillo, A. Qualitative assessment of EOB-GD-DTPA and Gd-BT-DO3A MR contrast studies in HCC patients and colorectal liver metastases. Infect. Agent Cancer 2019, 14, 40. [CrossRef] [PubMed]

24. Granata, V.; Fusco, R.; de Lutio di Castelguidone, E.; Avallone, A.; Palaia, R.; Delrio, P.; Tatangelo, F.; Botti, G.; Grassi, R.; Izzo, F.; et al. Diagnostic performance of gadoxetic acid-enhanced liver MRI versus multidetector CT in the assessment of colorectal liver metastases compared to hepatic resection. BMC Gastroenterol. 2019, 19, 129. [CrossRef] [PubMed]

25. Granata, V.; Fusco, R.; Avallone, A.; Catalano, O.; Piccirillo, M.; Palaia, R.; Nasti, G.; Petrillo, A.; Izzo, F. A radiologist's point of view in the presurgical and intraoperative setting of colorectal liver metastases. Future Oncol. 2018, 14, 2189-2206. [CrossRef]

26. Granata, V.; Fusco, R.; Catalano, O.; Avallone, A.; Palaia, R.; Botti, G.; Tatangelo, F.; Granata, F.; Cascella, M.; Izzo, F.; et al. Diagnostic accuracy of magnetic resonance, computed tomography and contrast enhanced ultrasound in radiological multimodality assessment of peribiliary liver metastases. PLoS ONE 2017, 12, e0179951. [CrossRef]

27. Granata, V.; Fusco, R.; Catalano, O.; Filice, S.; Amato, D.M.; Nasti, G.; Avallone, A.; Izzo, F.; Petrillo, A. Early Assessment of Colorectal Cancer Patients with Liver Metastases Treated with Antiangiogenic Drugs: The Role of Intravoxel Incoherent Motion in Diffusion-Weighted Imaging. PLoS ONE 2015, 10, e0142876. [CrossRef]

28. Van Griethuysen, J.J.M.; Fedorov, A.; Parmar, C.; Hosny, A.; Aucoin, N.; Narayan, V.; Beets-Tan, R.G.H.; Fillion-Robin, J.C.; Pieper, S.; Aerts, H.J.W.L. Computational Radiomics System to Decode the Radiographic Phenotype. Cancer Res. 2017, 77, e104-e107. [CrossRef]

29. Zwanenburg, A.; Vallières, M.; Abdalah, M.A.; Aerts, H.J.W.L.; Andrearczyk, V.; Apte, A.; Ashrafinia, S.; Bakas, S.; Beukinga, R.J.; Boellaard, R.; et al. The Image Biomarker Standardization Initiative: Standardized Quantitative Radiomics for High-Throughput Image-based Phenotyping. Radiology 2020, 295, 328-338. [CrossRef]

30. Fusco, R.; Sansone, M.; Filice, S.; Carone, G.; Amato, D.M.; Sansone, C.; Petrillo, A. Pattern Recognition Approaches for Breast Cancer DCE-MRI Classification: A Systematic Review. J. Med. Biol. Eng. 2016, 36, 449-459. [CrossRef] 
31. Beckers, R.C.J.; Trebeschi, S.; Maas, M.; Schnerr, R.S.; Sijmons, J.M.L.; Beets, G.L.; Houwers, J.B.; Beets-Tan, R.G.H.; Lambregts, D.M.J. CT texture analysis in colorectal liver metastases and the surrounding liver parenchyma and its potential as an imaging biomarker of disease aggressiveness, response and survival. Eur. J. Radiol. 2018, 102, 15-21. [CrossRef]

32. Andersen, I.R.; Thorup, K.; Andersen, M.B.; Olesen, R.; Mortensen, F.V.; Nielsen, D.T.; Rasmussen, F. Texture in the monitoring of regorafenib therapy in patients with colorectal liver metastases. Acta Radiol. 2019, 60, 1084-1093. [CrossRef] [PubMed]

33. Zhang, H.; Li, W.; Hu, F.; Sun, Y.; Hu, T.; Tong, T. MR texture analysis: Potential imaging biomarker for predicting the chemotherapeutic response of patients with colorectal liver metastases. Abdom. Radiol. 2018, 44, 65-71. [CrossRef]

34. Lubner, M.G.; Stabo, N.; Lubner, S.J.; del Rio, A.M.; Song, C.; Halberg, R.B.; Pickhardt, P.J. CT textural analysis of hepatic metastatic colorectal cancer: Pre-treatment tumor heterogeneity correlates with pathology and clinical outcomes. Abdom. Imaging 2015, 40, 2331-2337. [CrossRef] [PubMed]

35. Simpson, A.L.; Doussot, A.; Creasy, J.M.; Adams, L.B.; Allen, P.J.; DeMatteo, R.P.; Gönen, M.; Kemeny, N.E.; Kingham, T.P.; Shia, J.; et al. Computed Tomography Image Texture: A Noninvasive Prognostic Marker of Hepatic Recurrence After Hepatectomy for Metastatic Colorectal Cancer. Ann. Surg. Oncol. 2017, 24, 2482-2490. [CrossRef] [PubMed]

36. Ganeshan, B.; Miles, K.A.; Young, R.C.; Chatwin, C.R. Hepatic Enhancement in Colorectal Cancer: Texture Analysis Correlates with Hepatic Hemodynamics and Patient Survival. Acad. Radiol. 2007, 14, 1520-1530. [CrossRef]

37. Rahmim, A.; Bak-Fredslund, K.P.; Ashrafinia, S.; Lu, L.; Schmidtlein, C.; Subramaniam, R.M.; Morsing, A.; Keiding, S.; Horsager, J.; Munk, O.L. Prognostic modeling for patients with colorectal liver metastases incorporating FDG PET radiomic features. Eur. J. Radiol. 2019, 113, 101-109. [CrossRef]

38. Dercle, L.; Lu, L.; Schwartz, L.H.; Qian, M.; Tejpar, S.; Eggleton, P.; Zhao, B.; Piessevaux, H. Radiomics Response Signature for Identification of Metastatic Colorectal Cancer Sensitive to Therapies Targeting EGFR Pathway. J. Natl. Cancer Inst. 2020, 112, 902-912. [CrossRef]

39. Ravanelli, M.; Agazzi, G.M.; Tononcelli, E.; Roca, E.; Cabassa, P.; Baiocchi, G.L.; Berruti, A.; Maroldi, R.; Farina, D. Texture features of colorectal liver metastases on pretreatment contrast-enhanced CT may predict response and prognosis in patients treated with bevacizumab-containing chemotherapy: A pilot study including comparison with standard chemotherapy. Radiol. Med. 2019, 124, 877-886. [CrossRef]

40. Taghavi, M.; Staal, F.C.; Simões, R.; Hong, E.K.; Lambregts, D.M.; van der Heide, U.A.; Beets-Tan, R.G.; Maas, M. CT radiomics models are unable to predict new liver metastasis after successful thermal ablation of colorectal liver metastases. Acta Radiol. 2021, 17, 2841851211060437. [CrossRef]

41. Rizzetto, F.; Calderoni, F.; de Mattia, C.; Defeudis, A.; Giannini, V.; Mazzetti, S.; Vassallo, L.; Ghezzi, S.; Sartore-Bianchi, A.; Marsoni, S.; et al. Impact of inter-reader contouring variability on textural radiomics of colorectal liver metastases. Eur. Radiol. Exp. 2020, 4, 62. [CrossRef] [PubMed] 